\title{
Marcação, dispersão e capacidade de parasitismo de Cotesia flavipes (CAMERON) (HYMENOPTERA: BRACONIDAE)
}

Alan Carlos de Oliveira Castro ${ }^{1}$, Daniel Ferreira Caixeta ${ }^{2}$, Marco Tulio Pimenta Oliveira ${ }^{2}$, Maiara Geniffer dos Santos ${ }^{2}$, Rodrigo Fernandes de Souza ${ }^{2}$, Matheus Vinicius Abadia Ventura ${ }^{1}$

${ }^{1}$ Instituto Federal Goiano - IF Goiano, GO. ${ }^{2}$ Faculdade Evangélica de Goianésia, GO. E-mail: E-mail: alan.agrogsia@gmail.com

\section{Resumo}

O objetivo deste trabalho foi avaliar os aspectos biológicos de $C$. flavipes e sua marcação, além do desenvolvimento de uma metodologia para captura de adultos. Lagartas de $D$. saccharalis foram alimentadas com dieta artificial contendo os corantes SudanRed 7B e Solvent Blue 35 nas concentrações de 200 e 400 ppm, óleo vegetal a 200 e 400 ppm e sem óleo. Posteriormente, as lagartas foram parasitadas nos tratamentos supracitados. Avaliou-se o período entre o parasitismo e a emergência das larvas (ovolarval), a empupação, o peso das massas e das pupas, razão sexual, longevidade dos adultos e marcação. Após a definição do corante/concentração adequado para marcar adultos de $C$. flavipes, avaliou-se a capacidade de dispersão e parasitismo das fêmeas adultas em condições de campo. Utilizou-se o teste de Dunnett para as variáveis relacionadas ao desenvolvimento de larvas, pupas e adultos de $C$. flavipes e o procedimento de GLM para análise de variância e o LSMEANS para ajustar as medias em cada fator testado. SudanRed 7B na concentração de 200 ppm foi o melhor tratamento para marcar o parasitoide larval $C$. flavipes. Após, foi desenvolvida a metodologia para captura de $C$. flavipes, que associada à marcação, permitiu avaliar a capacidade de voo pela distância e captura desses indivíduos com precisão. Foi definida a armadilha de garrafa pet 600 como a melhor para avaliação de captura e parasitismo. A distância máxima de voo de indivíduos em campo foi de 17 metros, sendo que o maior número capturado foi até cinco metros. $80 \%$ das armadilhas com fêmeas capturadas foram parasitadas.

Palavras-chave: controle-biológico; voo; broca da cana-de-açúcar; corante lipossolúvel.

\section{Mark and dispersion of Cotesia flavipes parasitism capacity (CAMERON) (HYMENOPTERA: BRACONIDAE)}

\begin{abstract}
The objective of this study was to evaluate the biological aspects of $C$. flavipes your markup, and the development of a methodology for adult capture. Sugarcane borers fed on artificial diet containing the dyes SudanRed 7B and Solvent Blue 35 in concentrations of 200 and 400 ppm, vegetable oil at 200 and 400 ppm and without oil. Later, the caterpillars were parasitized in the above treatments. We evaluated the period between parasitism and larvae emergence, pupation, weight of the masses and pupae, sex ratio, adult longevity and marking. After the setting of the dye / marker suitable concentration for adult C. flavipes, we evaluated the dispersibility and parasitism of adult females under field conditions. We used the Dunnett test for the variables related to the development of larvae, pupae and adults of $C$. flavipes and the GLM procedure for analysis of variance and the LSMEANS to fit the media in each factor tested. Set up The SudanRed 7B dye at a concentration of $200 \mathrm{ppm}$ is the best dye / concentration to mark the larval parasitoid C. flavipes after developed the methodology for capturing $C$. flavipes, which associated with marking, allowed evaluating the flight capacity by distance and capture these individuals accurately. It was defined bottle trap 600 as the best pet for evaluation capture and parasitism. The maximum flight distance field in individuals was 17 meters, and the captured number was increased up to five meters. $80 \%$ of the traps with captured females were parasitized.
\end{abstract}

Keywords: biological control; flight; sugar cane borer; oil-soluble dyes. 


\section{Introdução}

A cultura da cana-de-açúcar é alvo de diversos problemas fitossanitários como pragas e doenças, e dentre os organismos mais prejudiciais à cultura ressalta-se a broca da canade-açúcar (Diatraea saccharalis (Fabricius, 1794) (Lepidoptera: Crambidae)) considerada uma das principais pragas da cultura (DINARDO-MIRANDA et al., 2012). Logo após a eclosão, as lagartas raspam o limbo foliar, e a partir do $2^{\circ}$ ínstar penetram a região do palmito por meio de galerias perfuradas nos colmos. Os principais sintomas observados são: morte do meristema apical, perda de peso, brotações e enraizamento laterais e quebra de entrenós pela ação do vento, além de redução de açúcar e álcool (MACEDO; BOTELHO, 1988; ROSSATO et al., 2013; RÔDAS et al., 2019).

A injúria provocada pelo inseto pode, indiretamente, favorecer a entrada de microrganismos como Colletotrichum spp. e Fusarium spp., que provocam o sintoma conhecido como podridão vermelha do colmo. A colonização desses patógenos na planta promove, principalmente, inversão da sacarose e redução da produção de álcool (LONG; HENSLEY, 1972; MACEDO; BOTELHO, 1988). Rossato et al. (2013) demonstraram que para cada $1 \%$ de intensidade de infestação podem ocorrer reduções de 8 a 19\% na produção de açúcar, além de aumentar a quantidade de fibra e compostos fenólicos no caldo, culminando na redução da qualidade do açúcar.

No Brasil, a principal ferramenta utilizada no controle de $D$. sccharalis é o parasitóide larval Cotesia flavipes (Cameron) (Hymenoptera: Braconidae). Esse inseto apresenta desenvolvimento completo e ciclo de vida de cerca de 20 dias. A fase larval tem três instares e ocorre dentro do hospedeiro, quando este está entre o terceiro e o quinto instar larval (BOTELHO; MACEDO, 2002). Ao final desse período as larvas saem do hospedeiro e tecem casulos para proteção das pupas.

Apesar de haver diversas informações à respeito do desenvolvimento e comportamento de parasitismo de $C$. flavipes, não existem dados consistentes a respeito da marcação e do voo desses indivíduos no estado de Goiás, em de São Paulo Volpe et al. (2009) comprova que uma alternativa viável seria o uso de técnicas de marcação de insetos com corante SudanRed B, por garantir que os inseto capturados sejam os mesmos que o liberados.

Sabe-se que a liberação e captura é uma das principais estratégias para estudar o voo dos insetos em condições de campo, desse modo, é possível determinar o raio de dispersão de um predador ou parasitoide em diversos agroecossistemas, além de viabilizar estudos sobre longevidade, crescimento, densidade e comportamento (CONRAD et al., 2002).

A marcação possibilita a realização de estudos de movimentação de insetos, além de avaliar seu potencial de parasitismo no campo, assim, os indivíduos capturados podem ser rastreados com mais facilidade. $O$ uso de corantes é simples e eficiente, e permite a marcação de diversos indivíduos a um custo baixo (OSTLIE et al., 1984). Após a marcação, é possível distinguir com facilidade os indivíduos liberados dos insetos selvagens, garantindo a distinção do inseto liberado até o momento da captura (SOUTHWOOD, 1992). Ressalta-se que o marcador ideal não pode afetar a longevidade e o comportamento do inseto, nem, tão pouco, o voo (FERNANDES, 2002).

Os corantes lipossolúveis são ferramentas importantíssimas nesse contexto, entretanto, faz-se necessário definir o tipo, cor e quantidade de corante necessária para marcar o inseto, sem prejudicá-lo (FERNANDES, 2002; CAIXETA, 2014). Assim, estudos relacionados à marcação, movimentação e capacidade de parasitismo do parasitoide C. flavipes em condições de campo são necessários para fomentar discussões que fortaleçam o maior programa de controle biológico do mundo. $\mathrm{O}$ objetivo do trabalho foi determinar o melhor corante/concentração para a marcação para avaliar a dispersão de $C$. flavipes.

\section{Material e Métodos}

\subsection{Marcação de C. flavipes}

Os ensaios foram conduzidos no Laboratório de Entomologia da Faculdade Evangélica de Goianésia, coordenadas $15^{\circ} 19^{\prime} \mathrm{S}$ $49^{\circ} 08^{\prime} \mathrm{O}$, em sala climatizada com temperatura de $26 \circ \mathrm{C}\left( \pm 2^{\circ} \mathrm{C}\right)$, umidade relativa de $60 \%( \pm 10 \%)$ e fotofase de 12 horas.

Folhas contendo posturas de $D$. saccharalis foram acondicionadas em folhas de papel sulfite em recipientes plásticos contendo 
algodão úmido para evitar o ressecamento dos ovos até a eclosão das larvas. Ao todo foram colocados cerca de 150 ovos em copos de $600 \mathrm{ml}$ com dieta artificial. Posteriormente, as lagartas foram transferidas, utilizando pincel, para copos de $100 \mathrm{ml}$ contendo diferentes tipos de dieta. Somente à partir do quarto ínstar as lagartas foram oferecidas para a oviposição de C.flavipes.

\subsection{Experimento 1}

Segundo Volpe et al. (2009), a dosagem com 100 ppm na dieta não é suficiente para a marcação de $C$. flavipes, desse modo, os tratamentos testados foram corante SudanRed 7B e Solvent Blue 35 nas concentrações de 200 e 400 ppm, além das dietas com óleo vegetal a 200 e $400 \mathrm{ppm}$ e a testemunha sem óleo, totalizando 7 tratamentos. Vinte e cinco dias depois, as lagartas marcadas e não marcadas foram parasitadas por $C$. flavipes e acondicionadas em seus respectivos copos até a empupação das larvas do parasitoide.

Soluções contendo os corantes lipossolúveis vermelho (Sudan Red 7B - C.I.26050) e azul (Solvent Blue 35 - C.I.61554) (Aldrich Company Inc.) foram incorporadas ao alimento oferecido às lagartas. Inicialmente, diluiu-se cinco $\mathrm{g}$ do corante em pó em $50 \mathrm{ml}$ de óleo de girassol (cultura não Bt). Para obtenção de 200 ppm, foram retirados dois $\mathrm{mL}$ desta solução contendo $0,2 \mathrm{~g}$ de corante e incorporados a um $\mathrm{L}$ de dieta (OSTLIE et al., 1984; VILARINHO et al., 2006). Os demais tratamentos foram obtidos a partir do mesmo princípio. A mistura foi homogeneizada durante um minuto e vertida em bandejas plásticas para resfriar em temperatura ambiente por duas horas. Em seguida, foi cortada em cubos de aproximadamente $2,5 \mathrm{~cm}$ e colocadas em copos de $100 \mathrm{ml}$.

O ensaio de marcação foi composto por sete tratamentos e dez repetições de três lagartas cada, adotando o delineamento inteiramente casualizado. A dieta utilizada foi a mesma adotada pelo laboratório de controle biológico da empresa Jalles Machado S/A, que utiliza a dieta modificada de King e Hartley (1985). Avaliou-se o período entre o parasitismo e a emergência das larvas (ovo-larval), a empupação, o peso das massas e individual, razão sexual e longevidade dos adultos. Os indivíduos foram considerados marcados quando o corante foi mantido até a fase adulta. Considerando o tamanho reduzido e a coloração escura, foi necessário espremê-los em papel A4, esse método de teste possibilitou avaliar a eficiência da marcação.

\subsection{Experimento 2}

\section{Armadilhas utilizadas para avaliar a captura e parasitismo de $C$. flavipes}

$\mathrm{Na}$ literatura não existem dados consistentes sobre ferramentas que permitam a captura de adultos de $C$. flavipes e avaliem o seu parasitismo. Desse modo, foi desenvolvida uma armadilha utilizando garrafa pet de água, de 600 $\mathrm{ml}$, cortada na parte superior, e utilizando a boca invertida para dentro. Visando reduzir o orifício de entrada e saída, utilizou-se mangueiras plásticas sobrepostas com 19, 7 e $5 \mathrm{~mm}$, que foram fixadas com cola quente a base de silicone para confeccionar um tipo de "funil" (Figura 2). $\mathrm{Na}$ face interna do funil foi aplicada graxa de engrenagens abertas (Acoplalub ${ }^{\circ}$ ) para evitar que os adultos de $C$. flavipes saíssem da armadilha. Essa graxa foi escolhida por não apresentar odor forte e por ser usada em fabricas alimentícias.

Para validação foi realizada seis repetições por cada fagoestimulante conforme descrito na tabela 2, a fim de identificar os melhores estímulos para captura. A versão final da armadilha possui três tipos estímulos, o primeiro é alimentar e consiste em um chumaço de algodão fixado à borda do funil, que recebeu caldo de cana; o segundo é olfativo, com fezes de lagartas de D.saccharalis em toda a entrada do funil; e o terceiro é a presença de 10 lagartas entre o $4^{\circ}$ e $5^{\circ}$ ínstar se alimentando de discos de cana com cerca de 1,5 de largura no interior da armadilha (Figura 1). Presentes em todas as armadilhas.

Figura 1. Descrição detalhada dos estímulos presentes na armadilha desenvolvida AC600.

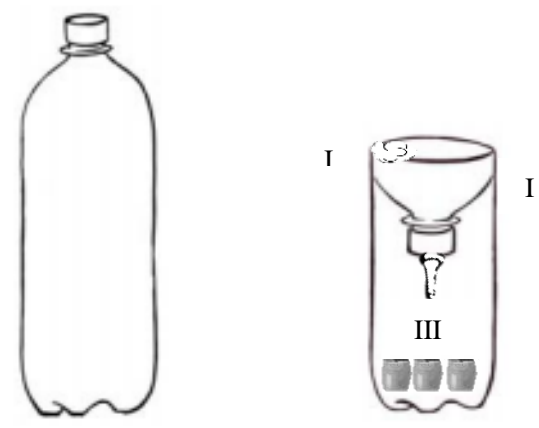

I - Chumaço de algodão fixado à borda do funil. II Fezes de lagartas de D.saccharalis em toda a entrada do funil. III - Lagartas entre o $4^{\circ}$ e $5^{\circ}$ ínstar se alimentando de discos de cana. 


\section{Liberação e captura da C. flavipes}

Os ensaios para avaliar a capacidade de dispersão de $C$. flavipes foram conduzidos em área comercial de cana-de-açúcar na Usina Jalles Machado S/A, fazenda Tapajós coordenadas $15^{\circ}$ $30^{\prime} \mathrm{S}, 48^{\circ} 58^{\prime} \mathrm{O}$, plantada com a variedade CTC 18 no ponto 1 e ponto 2 . A primeira liberação foi feita no dia 20/05/2015 as 11 horas e 20 minutos no ponto 1 , e às 17 horas no ponto 2 .

Os insetos foram liberados no ponto central e as armadilhas foram espaçadas a um metro entre si, até a distância máxima de 20 metros nos sentidos norte, sul, leste e oeste. As mesmas foram demarcadas com fita métrica e a ajuda de um GPS de navegação (Extrex 20,
GARMIN ). As armadilhas AC600 foram fixadas em colmos de cana com fita adesiva a cerca de um metro e meio do solo (Figura 2).

As armadilhas foram coletadas 72 horas após a liberação de adultos $C$. flavipes no campo. Posteriormente, foram levadas para o laboratório para quantificar os indivíduos capturados, marcados e selvagens (machos e fêmeas). As lagartas utilizadas como iscas foram colocadas em dietas por dez dias para a avaliação de parasitismo.

Figura 2. Armadilha utilizada nos experimentos e linha de armadilhas.

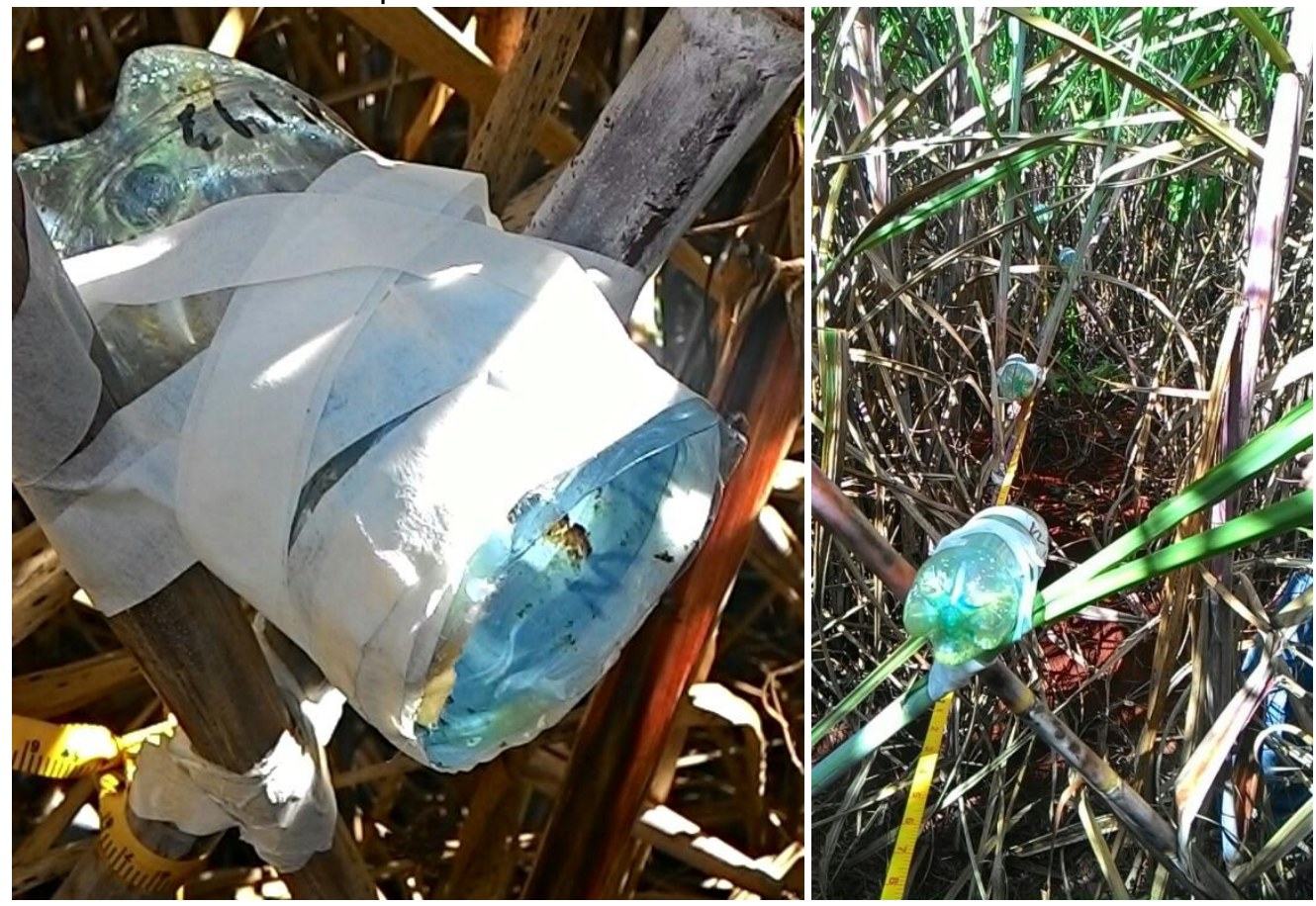

\section{Análise estatística}

Nos ensaios de marcação, consideraram-se os sete tratamentos como fatores qualitativos estruturados. Por isso, utilizou-se o teste de Dunnett para as variáveis relacionadas ao desenvolvimento de larvas, pupas e adultos de $C$. flavipes (BERTOLDO et al., 2008). Utilizou-se o procedimento GLM (General Linear Model), para análise de variância e o LSMEANS para ajustar as médias em cada fator testado, uma vez que ambos são indicados para análises com dados desbalanceados (SAS Institute 2004). Já no ensaio de liberação e captura, os insetos capturados foram separados em quatro grupos, para facilitar a separação por distância, sendo eles de 1 a $5 \mathrm{~m}$, de 6 a $10 \mathrm{~m}$, de 11 a $15 \mathrm{~m}$ e de 16 a $20 \mathrm{~m}$ para avaliar a capacidade de voo e utilizado o teste de media para validação dos dados coletados por grupo.

\section{Resultados e Discussão Marcação de $C$. flavipes}

Apenas corante vermelho SudanRed 7B na concentração de 400 ppm afetou a longevidade ovo-larval de $C$. flavipes $(p<0,0001)$, prolongando o período supracitado (Tabela 1 ).

Houve redução no peso das massas de pupas e na quantidade de pupas por massa $(p<0,05)$ nos tratamentos contendo os corantes azul Solvent Blue 35 a 200 e 400 ppm e vermelho 
SudanRed 7B a 400 ppm, demonstrando que ambos os corantes afetam $O$ inseto negativamente em concentrações mais elevadas. Ao avaliar peso de machos podemos identificar que $o$ peso foi afetado em todas as concentrações, exceto no tratamento com o corante vermelho SudanRed 7B. O peso das massas também foi afetado pelo corante vermelho SudanRed 7B a 200 ppm, entretanto, esse fenômeno não se confirmou no peso de fêmeas, nem no peso unitário das pupas, desse modo, acredita-se que esse tratamento não interferiu no peso de $C$. flavipes (Tabela 1 ).

Todos os corantes/concentrações reduziram a viabilidade das pupas $(p<0,0001)$, especialmente o corante azul Solvent Blue 35 a 400 ppm. Entretanto, esse fenômeno não foi observado na duração da fase de pupa, cuja média foi de 7,34 dias. 0 peso unitário das pupas também não foi alterado pelos corantes/concentrações, mas a razão sexual foi comprometida pelo corante azul Solvent Blue 35 a $400 \mathrm{ppm}$, prejudicando sua biologia. $(p<0,0001)$ (Tabela 1).

Não houve interferência do corante SudanRed 7B na mortalidade das fêmeas com 48 horas após a emergência, nem na longevidade dos adultos (Tabela 1). Desse modo, observa-se que 0 corante/concentração que menos interferiu na biologia de $C$. flavipes foi 0 SudanRed 7B a 200 ppm (Tabela 1).

Todas os corantes/concentrações foram eficientes na marcação dos de $C$. flavipes na fase adulta, modificando a coloração visceral desses organismos. Ao todo, foram testados 25 machos e fêmeas de cada tratamento no teste de marcação utilizando papel (Figura 2), e observouse que $100 \%$ dos adultos de C. flavipes foram marcados, independente do corante e concentração. A testemunha e os tratamentos contendo óleo se mantiveram com a coloração natural do inseto (Figura 2).

O corante SudanRed 7B na concentração de 200 ppm foi selecionado como o melhor corante/concentração para marcar o parasitóide larval $C$. flavipes, considerando sua influência sob parâmetros biológicos avaliados e alta eficiência de marcação. Os demais tratamentos se tornaram inviáveis em função da alta concentração do corante, ou devido a uma maior influência sobre o desenvolvimento de $C$. flavipes. 
Tabela 1. Efeito dos corantes e concentrações no desenvolvimento biológico de $C$. flavipes.

\begin{tabular}{|c|c|c|c|c|c|c|c|c|c|c|c|c|c|c|c|}
\hline \multirow{2}{*}{$\begin{array}{l}\text { Tratamento } \\
\text { Testemunha }\end{array}$} & \multicolumn{2}{|l|}{$\begin{array}{l}\text { Longevidade Ovo- } \\
\text { Larval (dias) }\end{array}$} & \multicolumn{3}{|c|}{ Peso de massas (g) } & \multicolumn{3}{|c|}{ Pupas/massa } & \multicolumn{2}{|c|}{ Peso de pupas (g) } & \multicolumn{2}{|r|}{$\begin{array}{l}\text { Inviabilidade } \\
\text { pupal (\%) }\end{array}$} & \multicolumn{3}{|c|}{$\begin{array}{c}\text { Longevidade } \\
\text { pupal } \\
\text { (unidade) } \\
\end{array}$} \\
\hline & \multicolumn{2}{|l|}{$12,48 \pm 0,22$} & \multicolumn{2}{|c|}{$0,0846 \pm 0,0088$} & & \multicolumn{2}{|c|}{$68,50 \pm 7,65$} & & \multicolumn{2}{|c|}{$0,001359 \pm 0,000174$} & & $15,21 \pm 3,94$ & & $7 \pm 0,42$ & \\
\hline Oleo a 400 ppm & $12,24 \pm 0,10$ & ns & \multicolumn{2}{|c|}{$0,0789 \pm 0,0065$} & ns 8 & \multicolumn{2}{|c|}{$82,20 \pm 5,75$} & ns & \multicolumn{2}{|c|}{$0,000955 \pm 0,00003$} & ns & $15,42 \pm 4,20$ & ns & $8 \pm 0,30$ & ns \\
\hline Vermelho 200 ppm & $12,23 \pm 0,09$ & ns & \multicolumn{2}{|c|}{$0,0588 \pm 0,0070$} & $*$ & \multicolumn{2}{|c|}{$50,90 \pm 5,75$} & ns & \multicolumn{2}{|c|}{$0,001202 \pm 0,000143$} & ns & $35,51 \pm 5,19$ & $*$ & $7,2 \pm 0,42$ & ns \\
\hline Vermelho 400 ppm & $13,41 \pm 0,17$ & $* *$ & \multicolumn{2}{|c|}{$0,0488 \pm 0,0046$} & ** 3 & \multicolumn{2}{|c|}{$35,80 \pm 1,52$} & $* *$ & \multicolumn{2}{|c|}{$0,001361 \pm 0,000111$} & ns & $36,10 \pm 6,27$ & $*$ & $7,6 \pm 0,27$ & ns \\
\hline \multirow[t]{2}{*}{ Azul a 400 ppm } & $12,33 \pm 0,12$ & $\mathrm{~ns}$ & & $0530 \pm 0,0031$ & ** 3 & $35,60 \pm 3$ & 3,31 & $* *$ & $0,001574 \pm 0$ &, 000131 & ns & $52,36 \pm 8,78$ & $* *$ & $7,9 \pm 0,28 \mathrm{~ns}$ & ns \\
\hline & Razão sexual & & & Peso de mach & $\mathrm{o}(\mathrm{g})$ & & Pes & so de $f$ & fêmea (g) & & (un & $\begin{array}{l}\text { de fêmeas } \\
\text { de) }\end{array}$ & & $\begin{array}{l}\text { sevidade fêm } \\
\text { (unidade) }\end{array}$ & \\
\hline Testemunha & $0,8391 \pm 0,023138$ & & & $0,000246 \pm 0$ & 000000 & & & 00024 & $4 \pm 0,000032$ & & $32 \pm$ & .8989 & & $2.76 \pm 0.0871$ & \\
\hline Óleo a200 ppm & $0,7945 \pm 0,040913$ & & ns & $0,000338 \pm 0$ & ,000018 & $8 \quad * *$ & 0,00 & 00364 & $4 \pm 0,0000004$ & $* *$ & $16 \pm$ & .4833 & ns & $2.84 \pm 0.0748$ & ns \\
\hline Azul a 200 ppm & $0,7299 \pm 0,043988$ & & ns & $0,000332 \pm 0$ & 000000 & 88 & & 00027 & $4 \pm 0,000034$ & ns & $12 \pm$ & .8989 & ns & $2.92 \pm 0.0553$ & ns \\
\hline Azul a 400 ppm & $0,4612 \pm 0,100355$ & & $* *$ & $0,000334 \pm 0$ & 000014 & $*$ & & 00024 & $6 \pm 0,000001$ & ns & $8 \pm 4$ & 8989 & $*$ & $2.92 \pm 0.0663$ & ns \\
\hline
\end{tabular}

Corante SudanRed 7B = Vermelho, corante azul Solvent Blue 35 = Azul. 
Figura 2. Avaliação da marcação artificial de Cotesia flavipes com corantes.
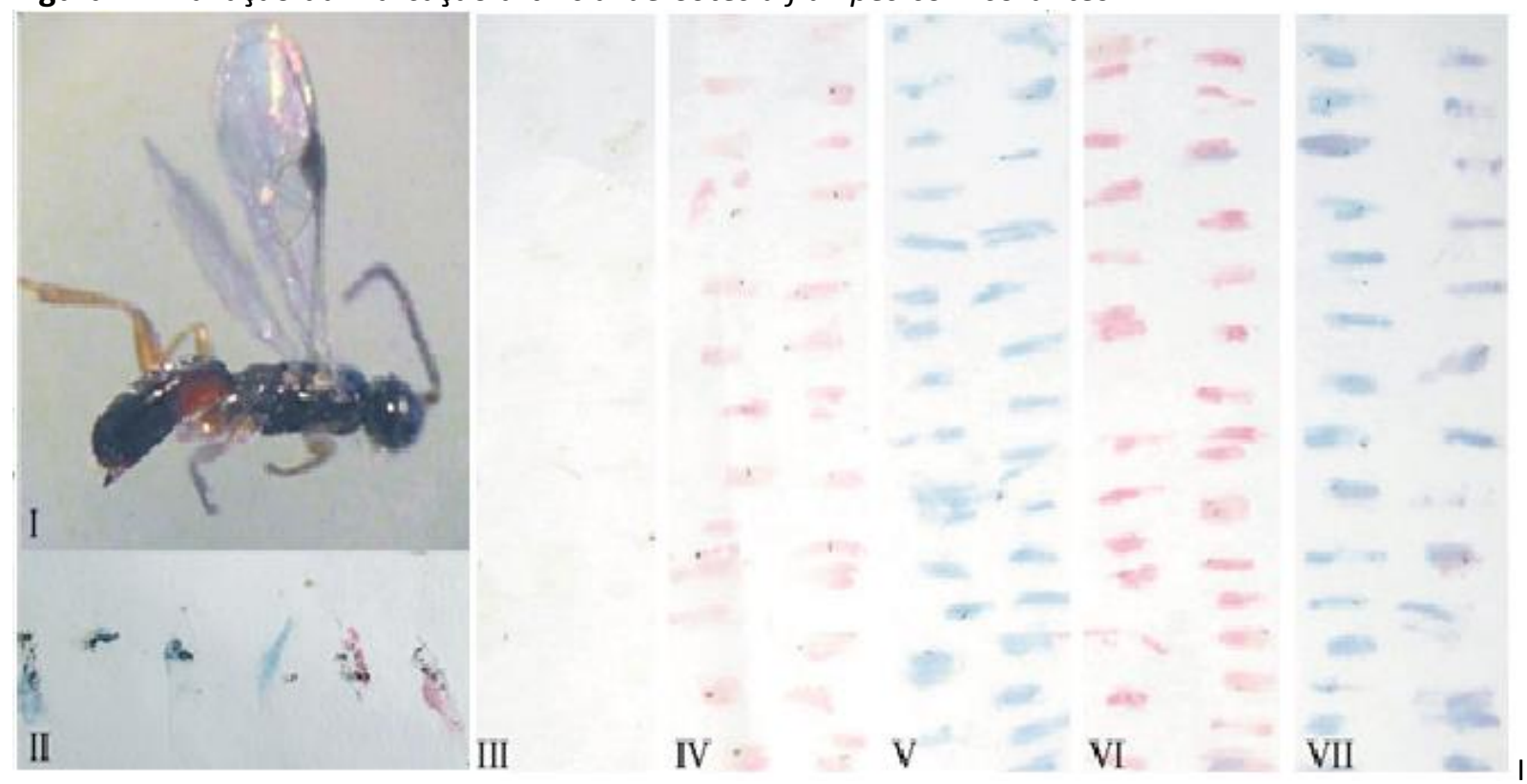

Adulto de $C$. flavipes marcado com o corante vermelho (Foto: cortesia de Maiara Geniffer Santos). II - esmagamento para visualização da marcação. Teste do papel com os tratamentos: III - testemunha e dietas com óleo, IV - corante vermelho SudanRed 7B a 200 ppm, V - corante azul Solvent Blue 35 a 200 ppm, VI - corante vermelho SudanRed 7B a 400 ppm, VII - corante azul Solvent Blue 35 a 400 ppm.

A aquisição dos corantes pelo parasitoide foi eficiente para todos os corantes/concentrações testados, à semelhança do que acontece com alguns lepidópteros (VILARINHO et al., 2006). Segundo Volpe et al. (2009), doses acima de $400 \mathrm{ppm}$ podem provocar efeitos negativos no desenvolvimento de $C$. flavipes, e isso também foi observado no presente trabalho, pois os aspectos biológicos dos insetos estão ligados à qualidade dos alimentos consumidos (WALDBAUER, 1968).

\section{Desenvolvimento da armadilha}

Foram realizados diversos testes para avaliar a eficiência de diferentes tipos de armadilhas e iscas (de forma empírica), é possível observar a evolução da captura em função do tipo de armadilha e das iscas utilizadas (Tabela 2). Em cada teste foram liberados cerca de 1500 indivíduos, e as armadilhas foram posicionadas a um metro de distância do ponto de liberação.

Tabela 2. Evolução da captura em diferentes tipos de armadilhas testados.

\begin{tabular}{lclc}
\multicolumn{1}{c}{ Armadilha: } & Alimento da isca & Fagoestimulante & Indivíduos Capturados \\
\hline PVC - $50 \mathrm{~mm}$ & Dieta artificial & Não possuía & 0 \\
Vidro com funil & Dieta artificial & Não possuía & 0 \\
Garrafa PET $600 \mathrm{ml}$ & Dieta artificial & Mel a 10\% & 25 \\
Garrafa PET $600 \mathrm{ml}$ & Dieta artificial & Mel a 10\% & 24 \\
Garrafa PET $600 \mathrm{ml}$ & Dieta artificial & Caldo de Cana & 17 \\
Garrafa PET $600 \mathrm{ml}$ & Dieta artificial & Caldo de Cana & 22 \\
Garrafa PET $600 \mathrm{ml}$ & Cana-de-açúcar & Caldo de cana & 85 \\
Garrafa PET $600 \mathrm{ml}$ & Cana-de-açúcar & Mel a 10\% & 74 \\
\hline
\end{tabular}

De acordo com Volpe et al. (2009), a coloração da garrafa pet não exerce influência alguma na captura dos indivíduos, tendo em vista que $C$. flavipes não e atraída preferencialmente por nenhum tipo de cor de bacias de armadilhas tipo Moericke. Assim foram feitos levantamentos e avaliações empíricas para determinar as melhores armadilhas e componentes. A 
armadilha AC600 proporcionou melhores resultados a respeito de captura e facilidade de confecção. o uso de alguns fagoestimulantes também aumentaram o índice de captura conforme a tabela 2. O protótipo que melhor resultados proporcionou foi $\mathrm{o}$ adotado no trabalho e o alimento usado nas armadilhas foi o de discos de cana.

\section{Dispersão de $C$. flavipes}

Duas liberações de adultos de $C$. flavipes foram realizadas simultaneamente, onde foram liberados aproximadamente 8850 indivíduos distribuídos em 27 copos de polietileno contendo cerca de 328 indivíduos por ponto. Foi estimado que $7,8 \%$ tenham morrido no local de liberação. A estimativa foi feita através da contagem de indivíduos que se encontraram mortos no copo após a liberação.

Após a coleta e avaliação do conteúdo das armadilhas, constatou-se que houve captura de até 16 indivíduos marcados em um único ponto. No ensaio 1 foram capturados $0,47 \%$ dos indivíduos liberados, e no ensaio 2 cerca de $0,58 \%$. Ao todo, foram recuperados 87 indivíduos marcados, e $87 \%$ foram capturadas até 4 metros. A distância máxima de voo de indivíduos marcados no campo foi de 17 metros, encontrados no grupo A. Os insetos capturados foram separados em quatro grupos para avaliar a capacidade de voo: A) de 1 a $5 \mathrm{~m}$; B) de 6 a $10 \mathrm{~m}$; C) de 11 a $15 \mathrm{~m}$; e D) de 16 a $20 \mathrm{~m}$.

A eficiência da armadilha em reter fêmeas após o parasitismo foi de $95 \%$, e houve parasitismo em $80 \%$ das fêmeas capturadas nas armadilhas. Vinte e cinco \% das 160 armadilhas deixadas no campo tiveram as lagartas parasitadas, sendo que nove foram parasitadas por fêmeas selvagens (22,5\%), 23 por fêmeas marcadas $(57,5 \%)$, seis por fêmeas selvagens ou marcadas, uma vez que o responsável pelo parasitismo não foi identificado (15\%), e duas por fêmeas que conseguiram escapar (5\%). Avaliadas através do número de insetos marcados.

Observou-se que $87,3 \%$ das fêmeas marcadas foram capturadas até cinco metros de distância do ponto de liberação, de seis a dez metros foram capturados $10,3 \%$, de onze a 15 metros $1,14 \%$, e de 15 a 20 metros $1,14 \%$. Ao comparar esses grupos, pôde-se observar um maior número de indivíduos nas cinco primeiras armadilhas, grupo $A(p<0,0001)$.

Até então, os métodos mais utilizados para avaliação de voo e parasitismo de $C$. flavipes consistem no uso de lagartas sentinelas (WHITE et al., 2004; WIEDENMANN; SMITH JR., 2006; VOLPE, 2009), entretanto, esse tipo de método exige avaliação populacional prévia para mensurar a presença de indivíduos selvagens na área. Outro problema é a rastreabilidade dos indivíduos liberados, que podem parasitar mais de uma armadilha. Esses problemas podem ser sanados com a utilização da armadilha desenvolvida nesse trabalho, pois reteve $95 \%$ das fêmeas após o parasitismo, que, associada à marcação desenvolvida, permite avaliar a capacidade de voo desses indivíduos com precisão.

\section{Conclusões}

1. O corante SudanRed 7B na concentração de 200 ppm é o melhor corante/concentração para marcar o parasitoide larval $C$. flavipes,

2. A armadilha AC600 se mostrou eficiente para captura de $C$. flavipes, associada à marcação desenvolvida, permite avaliar a capacidade de voo pela distância e captura desses indivíduos com precisão.

3. A armadilha AC600 é a melhor para avaliação de captura e parasitismo disponível na literatura até o presente momento.

4. A distância máxima de voo de indivíduos em campo foi de 17 metros, sendo que o maior número capturado foi até cinco metros.

5. Por meio dos resultados obtidos, torna se viável determinar a capacidade de voo e parasitismo de $C$. flavipes em campo, visando auxiliar a tomada de decisão quanto a sua liberação em campo.

\section{Referências}

BERTOLDO, J. G.; COIMBRA, J. L. M.; GUIDOLIN, A. F.; MIQUELOTO, A.; TOALDO, D. Use or abuse in mean comparison tests: scientific or empiric knowledge?. Ciência Rural, v. 38, n. 4, p. 11451148, 2008. https://doi.org/10.1590/S0103$\underline{84782008000400039}$

BOTELHO, P. S. M; MACEDO N. Cotesia flavipes para o controle de Diatraea saccharalis. In: PARRA, J. R. P.; BOTELHO, P. S. M.; CORRÊAFERREIRA, B. S.; BENTO, J. M. S. (Ed.). Controle biológico no Brasil: parasitóides e predadores. São Paulo: Manole, 2002. p. 477-494.

CAIXETA, D. F. Marcação, voo tutorado e dispersão de Anticarsia gemmatalis (Hübner) (Lepidoptera: Erebidae) e Chrysodeixis includens 
(Walker) (Lepidoptera: Noctuidae). 2014. $70 \mathrm{f}$. Tese (Doutorado em Agronomia) - Faculdade de Ciências Agrárias e Veterinárias, Universidade Estadual Paulista, Jaboticabal, 2014.

CONRAD, K. F.; WILLSON, K. H.; WHITFIELD, K.; HARVEY, I. F. J.; CHRIS. THOMAS; THOMAS N. SHERRATT. "Characteristics of dispersing Ischnura elegans and Coenagrion puella (Odonata): age, sex, size, morph and ectoparasitism."Ecography, v. 25, n. 4, p. 439-445, 2002. https://doi.org/10.1034/j.1600$\underline{0587.2002 .250406 . x}$

DINARDO-MIRANDA, L. L.; ANJOS, I. A. D.; COSTA, V. P. D.; FRACASSO, J. V. Resistance of sugarcane cultivars to Diatraea saccharalis. Pesquisa Agropecuária Brasileira, v. 47, n. 1, p. 1-7, 2012. https://doi.org/10.1590/S0100$\underline{\text { 204X2012000100001 }}$

FERNANDES, O. A. O uso de marcadores no controle biológico. In: PARRA, J. R. P.; BOTELHO, P. S. M.; CORRÊA-FERREIRA, B. S.; BENTO, J. M. S. (Ed.). Controle biológico no Brasil: parasitóides e predadores. São Paulo: Manole, 2002. p. 115121.

KING, E. G; HARTLEY, G. G. Diatraea saccharalis. In: SINGH, P.; MOORE, R. F. (Eds.). Handbook of insect rearing. New York: Elsevier, 1985. p. 265270.

LONG, W. H.; HENSLEY, S. D. Insect pests of sugar cane. Annual review of entomology, v. 17, n. 1, p. 149-176, 1972.

https://doi.org/10.1146/annurev.en.17.010172.0 $\underline{01053}$

MACEDO, N.; BOTELHO, P. S. M. Controle integrado da broca da cana-de-açúcar, Diatraea saccharalis (Fabr., 1794) (Lepidoptera: Pyralidae). Brasil Açucareiro, v. 106, n. 2, p. 2-14, 1988.

OSTLIE, K. R.; HEIN, G. L.; HIGLEY, L. G.; KASTER, L. V.; SHOWERS, W. B. "European corn borer (Lepidoptera: Pyralidae) development, larval survival, and adult vigor on meridic diets containing marker dyes." Journal of economic entomology, v. 77, n. 1, p. 118-120, 1984. https://doi.org/10.1093/jee/77.1.118
RÔDAS, P. L.; OLIVEIRA, H. N.; GLAESER, D. F. Liberação do parasitoide Cotesia flavipes (Hymenoptera: Braconidae) em Diatraea saccharalis (Lepidoptera: Crambidae) na cana-deaçúcar. Interciencia, v. 44, n. 5, p. 287-290, 2019.

ROSSATO, J. S. R.; COSTA, A.; MADALENO, G. H.; MUTTON, L. L; HIGLEY, L. G.; FERNANDES, O. A characterization and impact of the sugarcane borer on sugarcane yield and quality. Agronomy Journal, v. 105, n. 3, p. 643-648, 2013.

SOUTHWOOD, T. R. E. Ecological methods: with particular reference to the study of insect populations. London: Chapman \& Hall, 1992. 524 p.

VILARINHO, E. C.; FERNANDES, O A; OMOTO, C; HUNT, T. E. Oil-Soluble Dyes for Marking Spodoptera frugiperda (Lepidoptera: Noctuidae). Journal of Economic Entomology, Lanhan, v. 99, n. 6, p. 2110-2115, 2006.

https://doi.org/10.1093/jee/99.6.2110

VOLPE, H. X. L., A. M. VACARI, A. C. P. VEIGA, S. R. VIEL, S. A. DE BORTOLI. "Effect of Sudan Red B Dye in the mark and development of Diatraea saccharalis (Fabricius, 1794) (Lepidoptera: Crambidae) and Cotesia flavipes (Cameron, 1891) (Hymenoptera: Braconidae)." Boletín de Sanidad Vegetal, Plagas, v. 35, n. 2, p. 239-247, 2009.

VOLPE, H. X. L. Distribuição espacial do parasitismo de Cotesia flavipis (Cameron, 1891) (Hymenoptera: Braconidae) em cana-de-açucar. 2009. 67 f. Dissertação (Mestrado em Agronomia) - Faculdade de Ciências Agrarias e Veterinárias, Universidade Estadual Paulista, Jaboticabal, 2009.

WALDBAUER, G. P. The consumption and utilization of food by insects. Advances in insect physiology, v. 5, p. 229-288, 1968. https://doi.org/10.1016/S0065-2806(08)60230-1

WHITE, W. H.; REAGAN, T. E.; SMITH, J. W.; SALAZAR, J. A. Refuge releases of Cotesia flavipes (Hymenoptera: Braconidae) into the Louisiana sugarcane ecosystem. Environmental entomology, v. 33, n. 3, p. 627-632, 2004. https://doi.org/10.1603/0046-225X-33.3.627

WIEDENMANN, R. N.; SMITH JR, J. W. Use of ablated stalks to assess field rates of parasitism of Diatraea saccharalis (Lepidoptera: Crambidae) by 
Cotesia flavipes (Hymenoptera:

Braconidae). Biocontrol Science and Technology,

v. $16, \quad$ n. $1, \quad$ p. $37-48,2006$.

https://doi.org/10.1080/09583150500188056 